\title{
Unsteady Peristaltic Flow of Micro-Polar Fluid in a Finite Channel
}

\author{
Sanjay Kumar Pandey and Dharmendra Tripathi \\ Department of Applied Mathematics, Institute of Technology, Banaras Hindu University, \\ Varanasi-221005, India \\ Reprint requests to S.K. P; E-mail: skpandey.apm@itbhu.ac.in \\ or D. T.; E-mail: dtripathi.rs.apm@itbhu.ac.in
}

Z. Naturforsch. 66a, 181 - 192 (2011); received January 15, 2010 / revised September 17, 2010

This is an attempt to model an unsteady peristaltic flow of micro-polar fluid in a channel of finite length. The channel is subjected to progressive sinusoidal waves that help the walls contract and relax but not expand beyond the natural boundary. It is found that the coupling number increases pressure along the entire length of the channel, while the micro-polar parameter decreases it. The coupling number increases the efficiency; while the micro-polar parameter decreases it. The reflux region is found to increase with the coupling number. One significant difference between integral and non-integral number of waves in the train propagating along the channel walls is that the peaks of pressure are identical in the integral case while the peaks are different in the non-integral case.

Key words: Peristalsis; Oesophagus; Micro-Polar Fluid; Mechanical Efficiency; Reflux; Trapping.

\section{Introduction}

Eringen [1] formulated a concentrated suspension of non-deformable neutrally buoyant rigid particles in a viscous medium by duly considering the effect of individual particle in the flow. In fact, the effects are microrotational and micro-inertial. He termed the formulated fluid as micro-polar. Some physiological fluids are of this nature. Blood has some suspension of red blood corpuscles (RBC), white blood corpuscles (WBC) etc. in plasma; cervical mucus is a suspension of macromolecules in water-like liquid; and semi digested food is a mixture of liquid and solid particles. Some food materials may be viewed as a micro-polar fluid.

Devi and Devanathan [2] investigated the peristaltic transport of a micro-polar fluid in a cylindrical tube with a sinusoidal wave of small amplitude. Philip and Chandra [3] worked on the peristaltic transport of a simple micro-polar fluid which accounts for micro rotation and micro stretching of the particles contained in a small volume element, using long wavelength approximation. Bhargava et al. [4] modelled the peristaltic flow of blood through stenosed arteries by considering blood as micro-polar fluid and arteries as porous channel. Srinivasacharya et al. [5] studied different micro-polar properties; Hayat et al. [6] investigated the effects of different wave forms; Muthu et al. [7] studied wall properties; whereas Hayat and Ali [8] reported effects of an endoscope.

All the studies cited above have been carried out for an infinite tube with the walls contracting and dilating as well. However, the oesophagus is finite in length in comparison to its radius. Its wall undergoes contraction and relaxation but no expansion beyond the stationary boundary. Hence, none of the studies mentioned above is suitable for swallowing of a micro-polar fluid in oesophagus. Further, the pressure distribution along the oesophageal length, i. e., oesopageal manometry, is of prime importance, which has been often neglected in previous studies.

Dodds [9] and Ren et al. [10] carried out experimental investigations in this direction for different viscosities and bolus-volumes. Li and Brasseur [11] have theoretically worked on it for Newtonian fluids considering finite length tubes, and focused the study on both local and global dynamics. The issue of local dynamics such as spatial-temporal variations in local stresses in terms of the motility and efficacy of the transport process was raised by Brasseur and Dodds [12]. They found close resemblance with the experimental findings of Dodds [9] and Ren et al. [10]. Misra and Pandey [13] who investigated similar phenomena for power-law fluids came to similar conclusions, although they didn't discuss the experimental findings. Pandey and Tripathi $[14,15]$ have recently worked for mag- 


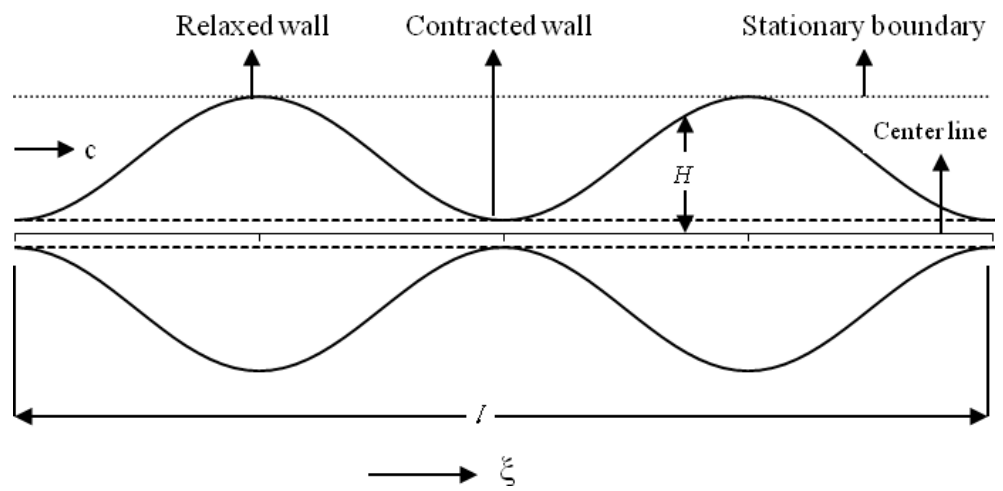

Fig. 1. Diagram, based on (7), represents the propagation of a progressive transverse wave along the walls of the channel containing fluid, which undergoes contraction and relaxation but no expansion beyond the boundary. neto hydro dynamic (MHD) and Maxwell fluids, respectively.

With a mission to investigate the transportation of physiological fluids through vessels, we have tried to model peristaltic transport in a channel of finite length. Physiological fluids are taken as micro-polar fluids. This model may be applied to transportation of food bolus in oesophagus, blood flow in aorta, and flow of semi-digested food in duodenum. From engineering point of view, this model may also be applied to study the flow of micro-polar fluids through a mechanical peristaltic pump. We have also examined here the case of propagation of a non-integral number of waves along the channel walls, which are inherent characteristics of finite length vessels.

\section{Mathematical Model}

We consider the flow of a micro-polar fluid in a channel of length $\tilde{l}$. The equations governing the motion of an incompressible micro-polar fluid in the absence of body forces and body couple and under the following dimensionless transformations:

$$
\begin{aligned}
& \xi=\frac{\tilde{\xi}}{\lambda}, \quad \eta=\frac{\tilde{\eta}}{a}, \quad t=\frac{c \tilde{t}}{\lambda}, \quad u=\frac{\tilde{u}}{c}, \quad v=\frac{\tilde{v}}{c \delta}, \\
& w=\frac{a \tilde{w}}{c}, \quad H=\frac{\tilde{H}}{a}, \quad l=\frac{\tilde{l}}{\lambda}, \quad \phi=\frac{\tilde{\phi}}{a}, \\
& \sigma=\frac{\tilde{\sigma}}{a^{2}}, \quad p=\frac{\tilde{p} a^{2}}{\mu c \lambda}, \quad \operatorname{Re}=\frac{\rho c a \delta}{\mu}, \quad Q=\frac{\tilde{Q}}{a c},
\end{aligned}
$$

are reduced to the dimensionless form given by

$$
\begin{aligned}
& \frac{\partial p}{\partial \xi}=\frac{1}{1-N}\left\{N \frac{\partial w}{\partial \eta}+\frac{\partial^{2} u}{\partial \eta^{2}}\right\}, \\
& \frac{\partial p}{\partial \eta}=0,
\end{aligned}
$$

$$
\begin{aligned}
& \frac{\partial^{2} w}{\partial \eta^{2}}=\frac{M^{2}}{2-N}\left(2 w+\frac{\partial u}{\partial \eta}\right), \\
& \frac{\partial u}{\partial \xi}+\frac{\partial v}{\partial \eta}=0,
\end{aligned}
$$

under long wavelength and low Reynolds number approximations. Here, $\tilde{H}, \tilde{\xi}, \tilde{\eta}, \tilde{t}, a, \tilde{\phi}, \lambda, c, \tilde{u}, \tilde{v}, \tilde{w}$, $\rho, \tilde{\sigma}, \operatorname{Re}$, and $\tilde{Q}$, represent transverse displacement of the wall, axial and transverse coordinates, time, half width of the channel, amplitude of the wave, wavelength, wave velocity, axial velocity, transverse velocity, micro-polar vector, fluid density, the microgyration parameter, the Reynolds number, and volume flow rate, respectively. The symbols without ' $\sim$ ' are the corresponding parameters in the dimensionless form. Moreover, $N=k /(\mu+k)$ is the coupling number, a measure of particle coupling with its surroundings $(0 \leq N \leq 1), M=\sqrt{a^{2} k(2 \mu+k) / \gamma(\mu+k)}$ is the micro-polar parameter, and $\alpha, \beta$ do not appear in the governing equation as the micro-rotation vector is solenoidal. The parameters $\mu, k, \alpha, \beta, \gamma$ are material constants and satisfy the following conditions:

$$
\begin{aligned}
& 2 \mu+k \geq 0, \quad k \geq 0, \\
& 3 \alpha+\beta+\gamma \geq 0, \quad \gamma \geq|\beta|,
\end{aligned}
$$

where $\delta=a / \lambda$ is the wave number. In the limiting case $k \rightarrow 0$, i.e., $N \rightarrow 0$, the governing equations for the micro-polar fluid reduce to the governing equations for a Newtonian fluid.

Oscillations are caused on the walls of the channel by propagating continuous contraction waves (Fig. 1) given in dimensionless form as

$$
H(\xi, t)=1-\phi \cos ^{2} \pi(\xi-t) .
$$

The boundary conditions, we shall apply, are

$$
\left.u(\xi, t)\right|_{\eta=H}=0,\left.\quad v(\xi, t)\right|_{\eta=H}=\frac{\partial H}{\partial t}
$$




$$
\begin{aligned}
& \left.v(\xi, t)\right|_{\eta=0}=0,\left.\quad \frac{\partial u(\xi, t)}{\partial \eta}\right|_{\eta=0}=0, \\
& \left.w(\xi, t)\right|_{\eta=0}=0,\left.\quad w(\xi, t)\right|_{\eta=H}=0 .
\end{aligned}
$$

Integrating (2) once with respect to $\eta$, we get

$$
\frac{\partial u}{\partial \eta}=(1-N) \frac{\partial p}{\partial \xi} \eta-N w+C
$$

where $C$ is a function, independent of $\eta$, to be evaluated later.

Further, integrating (4) twice with respect to $\eta$, and also using (10) and the boundary conditions (9), the micro-polar vector is obtained as

$$
\begin{aligned}
w= & \frac{1-N}{2-M} \frac{\partial p}{\partial \xi}\left\{\frac{H \sinh (M \eta)}{\sinh (M H)}-\eta\right\} \\
& +\frac{C}{2-N}\{\sinh (M \eta)+\sinh (M(H-\eta))-1\} .
\end{aligned}
$$

Then, using the fourth boundary condition of (8), (10) and (11) become

$$
\begin{aligned}
& \frac{\partial u}{\partial \eta}= \\
& (1-N) \frac{\partial p}{\partial \xi}\left[\eta-\frac{N}{2-N}\left\{\frac{H \sinh (M \eta)}{\sinh (M H)}-\eta\right\}\right], \\
& w=\frac{1-N}{2-N} \frac{\partial p}{\partial \xi}\left\{\frac{H \sinh (M \eta)}{\sinh (M H)}-\eta\right\} ;
\end{aligned}
$$

and further integrating (12) and applying the no-slip condition of (8), the axial velocity is obtained as

$$
\begin{aligned}
u= & \frac{1-N}{2-N} \frac{\partial p}{\partial \xi}\left[\left(\eta^{2}-H^{2}\right)-\frac{N H}{M \sinh (M H)}\right. \\
& \cdot\{\cosh (M \eta)-\cosh (M H)\}] .
\end{aligned}
$$

The transverse velocity is derived from the continuity equation (5) by substituting $u$ from (14) and integrating it once with respect to $\eta$. The regularity condition, given in (8), determines the constant term and gives the transverse velocity as

$$
\begin{gathered}
v=\frac{1-N}{2-N}\left[\frac { \partial p } { \partial \xi } \frac { \partial H } { \partial \xi } \left\{2 \eta H+\frac{N}{M^{2}}\left\{\frac{\sinh (M \eta)}{\sinh (M H)}\right.\right.\right. \\
-M \eta \operatorname{coth}(M H)+H(M \sinh (M \eta) \cosh (M H) \\
\left.\left.\left.\cdot \operatorname{cosech}^{2}(M H)-M^{2} \eta \operatorname{cosech}^{2}(M H)\right)\right\}\right\} \\
-\frac{\partial^{2} p}{\partial \xi^{2}}\left\{\frac{\eta^{3}-3 H^{2} \eta}{3}-\frac{N H}{M^{2} \sinh (M H)}\right. \\
\cdot(\sinh (M \eta)-M \eta \cosh (M H))\} .
\end{gathered}
$$

We apply the transverse velocity of the wall, given in (8), on the transverse velocity in (15). That is further integrated with respect to $\xi$, to obtain the pressure gradient as

$$
\begin{aligned}
\frac{\partial p}{\partial \xi}= & \frac{3 M^{2}(2-N)}{1-N}\left[G(t)+\int_{0}^{\xi} \frac{\partial H}{\partial \xi} \mathrm{d} s\right] \\
& \cdot\left[2 M^{2} H^{3}+3 N H(1-M H \operatorname{coth}(M H))\right]^{-1} .
\end{aligned}
$$

Integrating it once again from 0 to $\xi$, the pressure difference is obtained as

$$
\begin{gathered}
p(\xi, t)-p(0, t)=\frac{3 M^{2}(2-N)}{1-N} \\
\cdot \int_{0}^{\xi} \frac{G(t)+\int_{0}^{s} \frac{\partial H}{\partial \xi} \mathrm{d} s_{1}}{2 M^{2} H^{3}+3 N H(1-M H \operatorname{coth}(M H))} \mathrm{d} s,
\end{gathered}
$$

in which by substituting $\xi=l$, the pressure difference between the inlet and the outlet of the channel is obtained as

$$
\begin{aligned}
& p(l, t)-p(0, t)=\frac{3 M^{2}(2-N)}{1-N} \\
& \cdot \int_{0}^{l} \frac{G(t)+\int_{0}^{\xi} \frac{\partial H}{\partial \xi} \mathrm{d} s}{2 M^{2} H^{3}+3 N H(1-M H \operatorname{coth}(M H))} \mathrm{d} \xi,
\end{aligned}
$$

where $G(t)$ is a function required to be evaluated. A simple manipulation of this evaluates $G(t)$ as

$$
\begin{aligned}
& G(t)=\left[\frac{1-N}{3 M^{2}(2-N)} \Delta p_{l}(t)\right. \\
& \left.-\int_{0}^{l} \frac{\int_{0}^{\xi} \frac{\partial H}{\partial t} \mathrm{~d} s}{2 M^{2} H^{3}+3 N H(1-M H \operatorname{coth}(M H))} \mathrm{d} \xi\right] \\
& \cdot\left[\int_{0}^{l} \frac{1}{2 M^{2} H^{3}+3 N H(1-M H \operatorname{coth}(M H))} \mathrm{d} \xi\right]^{-1},
\end{aligned}
$$

where $\Delta p_{l}(t)=p(l, t)-p(0, t)$ is the pressure difference between the inlet and the outlet of the channel.

The volume flow rate is defined as $Q(\xi, t)=$ $\int_{0}^{H} u \mathrm{~d} \eta$, which, on performing the integration, yields

$$
\begin{aligned}
& Q(\xi, t)= \\
& \frac{N-1}{2-N} \frac{\partial p}{\partial \xi}\left\{\frac{2 H^{3}}{3}+\frac{N H}{M^{2}}(1-M H \operatorname{coth}(M H))\right\} .
\end{aligned}
$$

The average volume flow rate can be given in terms of the flow rate in the wave frame as

$$
\bar{Q}=q+1-\frac{\phi}{2}=Q-H+1-\frac{\phi}{2} .
$$


The pressure difference, in terms of averaged volume flow rate, is formulated by integrating the pressure gradient obtained from (20) and (21) as

$$
\begin{aligned}
& p(\xi)-p(0)=\frac{3 M^{2}(2-N)}{N-1} \\
& \cdot \int_{0}^{\xi} \frac{\bar{Q}+H-1+\frac{\phi}{2}}{2 M^{2} H^{3}+3 N H(1-M H \operatorname{coth}(M H))} \mathrm{d} s .
\end{aligned}
$$

Finally, the local wall shear stress $\tau_{\mathrm{w}}=\left.(\partial u / \partial \eta)\right|_{\eta=H}$, by virtue of (12) and (16), is given by

$$
\tau_{\mathrm{w}}=\frac{3 M^{2}(2-N)\left\{G(t)+\int_{0}^{\xi} \frac{\partial H}{\partial t} \mathrm{~d} s\right\}}{2 H^{2} M^{2}+3 N(1-M H \operatorname{coth}(M H))} .
$$

\section{Mechanical Efficiency}

Mechanical efficiency (cf. [16]) is derived for a micro-polar fluid in a finite channel as

$$
E=\frac{\bar{Q} \Delta p_{1}}{\phi\left(I_{1}-\Delta p_{1}\right)},
$$

where $I_{1}=\int_{0}^{1}(\partial p / \partial \xi) \cos 2 \pi \xi \mathrm{d} \xi$ and $\Delta p_{1}$, the pressure difference across one wavelength, is given, in view of (22), by

$$
\begin{aligned}
& \Delta p_{1}=p(1)-p(0)=\frac{3 M^{2}(2-N)}{N-1} \\
& \cdot \int_{0}^{1} \frac{\bar{Q}-1+\frac{\phi}{2}+H}{2 M^{2} H^{3}+3 N H(1-M H \operatorname{coth}(M H))} \mathrm{d} \xi
\end{aligned}
$$

and $\bar{Q}_{0}$, the maximum flow rate, is obtained by virtue of (25) and for $\Delta p_{1}=0$ as

$$
\begin{aligned}
& \bar{Q}_{0}=1-\frac{\phi}{2} \\
& -\left[\int_{0}^{1} \frac{1}{2 M^{2} H^{2}+3 N(1-M H \operatorname{coth}(M H))} \mathrm{d} \xi\right] \\
& \cdot\left[\int_{0}^{1} \frac{1}{2 M^{2} H^{3}+3 N H(1-M H \operatorname{coth}(M H))} \mathrm{d} \xi\right]^{-1} .
\end{aligned}
$$

\section{Reflux Limit}

Reflux is an inherent phenomenon of peristaltic movement, which refers to the presence of fluid particles that move, on the average, in a direction opposite to the net flow in the close vicinity of the wall (cf. [16]).
For the two-dimensional case, the dimensional stream function, in the wave frame, is $\mathrm{d} \tilde{\psi}=\tilde{U} \mathrm{~d} \tilde{\zeta}-$ $\tilde{V} \mathrm{~d} \tilde{\zeta}$, where $\tilde{\psi}, \tilde{\zeta}, \tilde{\zeta}, \tilde{U}$, and $\tilde{V}$ are stream function, coordinates and velocity-components, respectively. Under the transformations, where the parameters on the left of the equality sign are in the wave frame and those on the right are in the laboratory frame, given by $\tilde{\zeta}=\tilde{\xi}-c \tilde{t}, \tilde{\zeta}=\tilde{\eta}, \tilde{U}=\tilde{u}-c, \tilde{V}=\tilde{v}, \tilde{q}=\tilde{Q}-c \tilde{H}$, we obtain stream function as

$$
\begin{gathered}
\psi=-\frac{\bar{Q}-1+\frac{\phi}{2}+H}{2 M^{2} H^{3}+3 N H(1-M H \operatorname{coth}(M H))} \\
\cdot\left\{M^{2}\left(\eta^{3}-3 H^{2} \eta\right)-\frac{3 N H}{\sinh (M H)}\right. \\
\cdot(\sinh (M \eta)-M \eta \cosh (M H))\}-\eta .
\end{gathered}
$$

The stream function at the wall, $\psi_{\mathrm{w}}$, is solved from (27) by substituting $\eta=H$. A simplification yields

$$
\psi_{\mathrm{w}}=\bar{Q}-1+\frac{\phi}{2} \text {. }
$$

Reflux flow rate $Q_{\psi}(\xi)$, associated with a particle at the position $\xi$, is given by

$$
Q_{\psi}=\psi+\eta(\psi, \xi),
$$

which, on averaging over one cycle, gives

$$
\bar{Q}_{\psi}=\psi+\int_{0}^{1} \eta(\psi, \xi) \mathrm{d} \xi
$$

Moreover, in order to evaluate the reflux limit, $\bar{Q}_{\psi}$ is expanded in a power series in terms of a small parameter $\varepsilon$ about the wall, where $\varepsilon\left(=\psi-\psi_{\mathrm{w}}\right)$ is subjected to the reflux condition $\bar{Q}_{\psi} / \bar{Q}>1$ as $\varepsilon \rightarrow 0$. The coefficient of the first two terms in the expansion of $\eta$ is obtained only for small values of $M$. Substituting the expansion $\eta=H+a_{1} \varepsilon+a_{2} \varepsilon^{2}+\cdots$ into (27), and using (28), we get

$$
\begin{gathered}
a_{1}=-1, \quad 3 M^{2}\left(\bar{Q}-1+\frac{\phi}{2}+H\right) \\
a_{2}=-\frac{1 M^{2} H^{2}+3 N(1-M H \operatorname{coth}(M H))}{2\left(1-\frac{M N H}{2 \sinh (M H)}\right) .}
\end{gathered}
$$

Using (31) and applying reflux condition in (30), we obtain the reflux limit as 

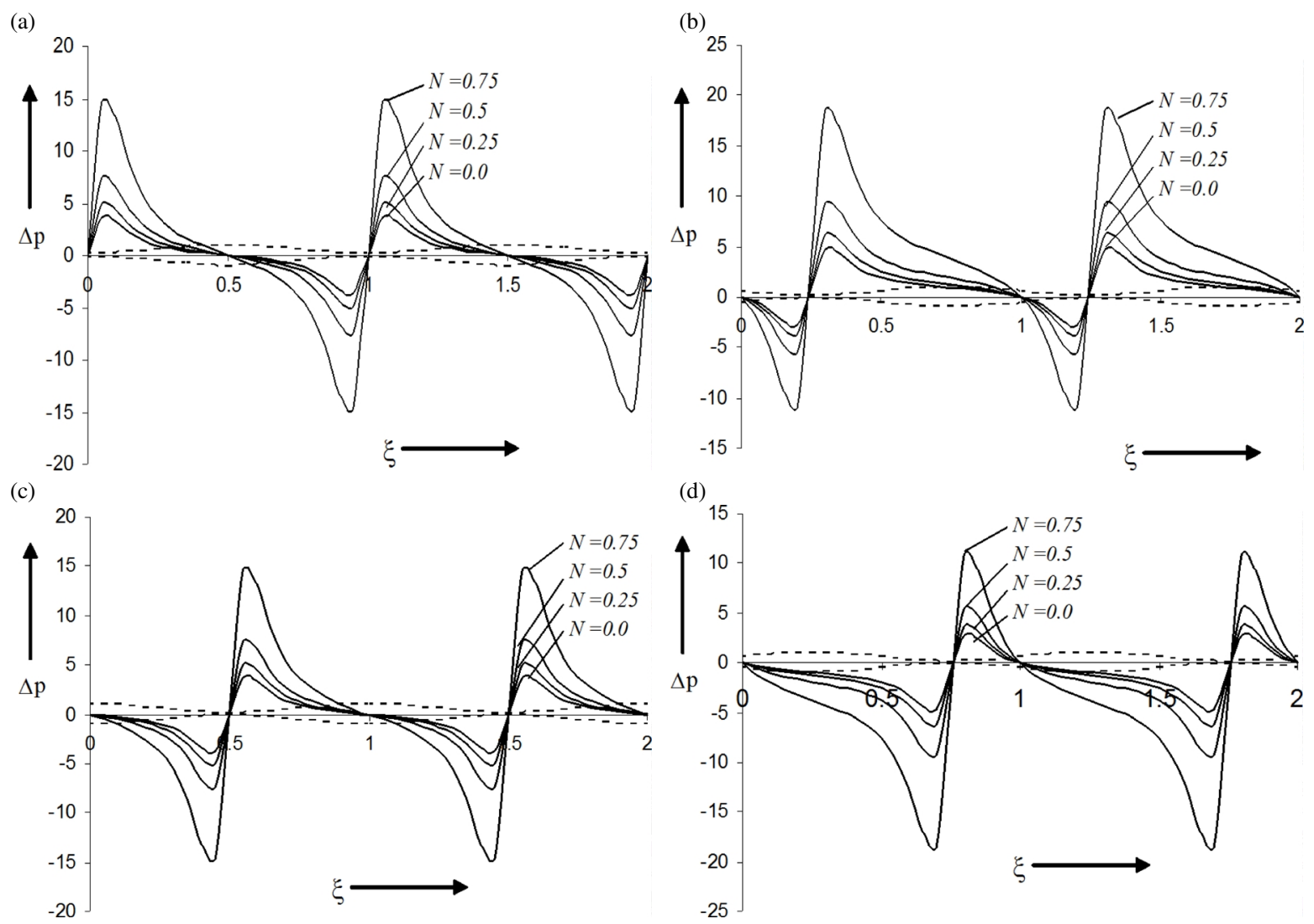

Fig. 2. Pressure vs. axial distance along the finite length channel at five time instants (a) $t=0.0, t=1.0$, (b) $t=0.25$, (c) $t=0.5$, (d) $t=0.75$. Dotted lines ( ...) represent the position of wave, whereas continuous lines (-) represent the pressure distribution for different coupling number $N$ and for $\phi=0.9, l=2.0, M=1.0$. The pressures at the two ends are kept as zero.

$$
\bar{Q}<1-\frac{\phi}{2}-\frac{\int_{0}^{1} \frac{H(2 \sinh (M H)-M N H)}{2 \sinh (M H)\left\{2 M^{2} H^{2}+3 N(1-M H \operatorname{coth}(M H))\right\}} \mathrm{d} \xi}{\int_{0}^{1} \frac{2 \sinh (M H)-M N H}{2 \sinh (M H)\left\{2 M^{2} H^{2}+3 N(1-M H \operatorname{coth}(M H))\right\}} \mathrm{d} \xi} .
$$

\section{Numerical Results and Discussion}

\subsection{Numerical Aspects}

A thorough examination of the effects of coupling number and micro-polar parameter, which together determine the micro-polar characteristic of the fluid on the flow pattern, is carried out by computer simulation. The case, we take up here, is that of free pumping, i.e., the case when the pressure across the two ends of the channel are zero. We write programs in $\mathrm{C}$ and observe graphs drawn based on the computational results.

First of all, we perform temporal examination of the pressure distribution along the length at various in- stants, i. e., for discrete values of $t$ in the range $0.0-$ 1.0 (Fig. 2). At $t=0$ (Fig. 2a), it is observed that the pressure tends to rise sharply at the inlet, reaches some peak, then falls at a lower rate to zero at the middle of the bolus, comes down further to a lower trough, and finally rises sharply to meet the leading end of the bolus. The same distribution is repeated for the next bolus. After one fourth of the periodic cycle (Fig. 2b), the bolus has moved ahead and a trailing bolus is on the way to entry. The graphs for higher values of $t$ represent a systematic progress of the boluses in the channel. Eventually, at the time $t=1$, which represents the completion of one period, the pressure distribution re- 
(a)

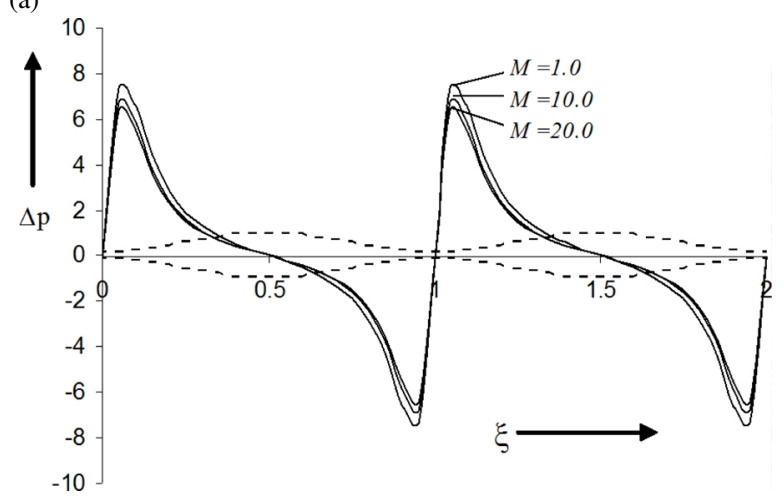

(c)

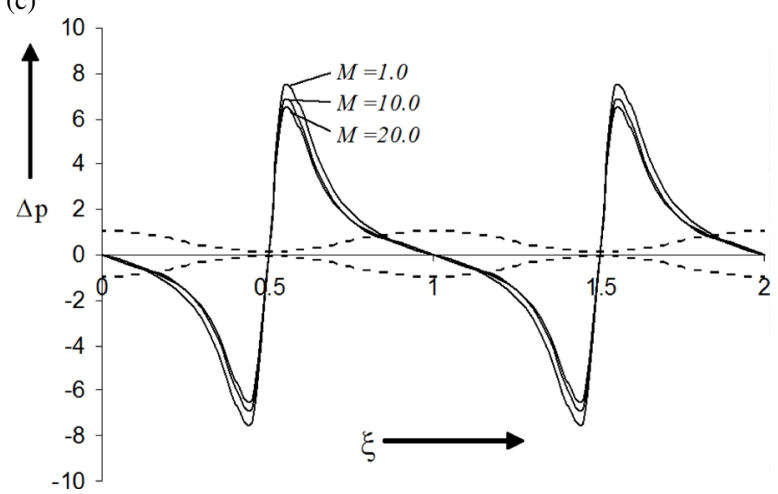

(b)

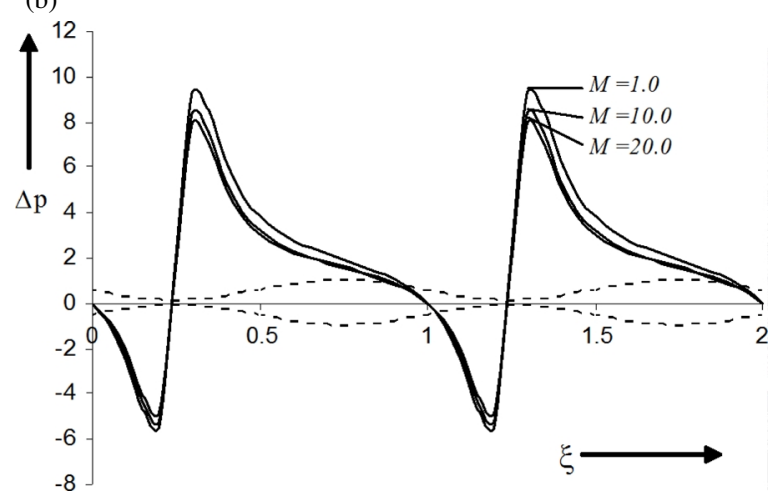

(d)

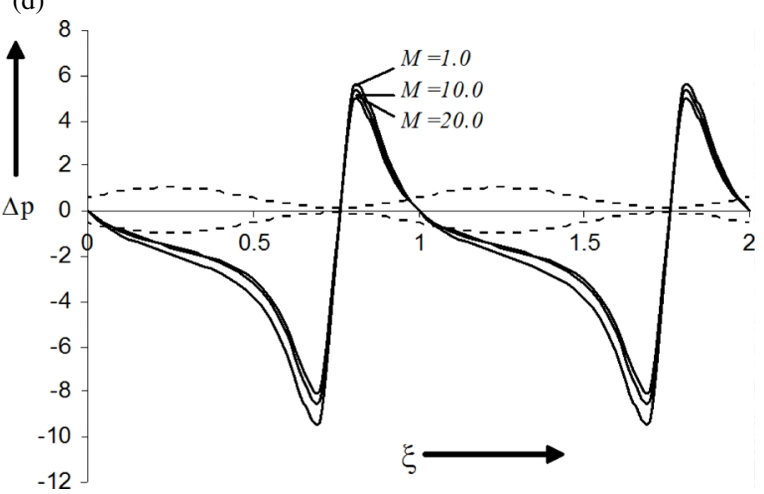

Fig. 3. Pressure vs. axial distance along the finite length channel at five time instants (a) $t=0.0, t=1.0$, (b) $t=0.25$, (c) $t=0.5$, (d) $t=0.75$. Dotted lines ( $\cdots$ ) represent the position of wave, whereas continuous lines $(-)$ represent the pressure distribution for different micro-polar parameter $M$ and for $\phi=0.9, l=2.0, N=0.50$. The pressures at the two ends are kept as zero.

sembles that at $t=0$ : this indicates that a new cycle is ready to set out (Fig. 2a).

It is further observed that the pressure distribution along the length of the channel for micro-polar fluid shows qualitative similarity with Newtonian fluid (Fig. 2) which is a special case, i. e., $N \rightarrow 0$.

It also reveals that as the coupling effect parameter $N$ increases, pressure gradient as well as pressure along the length of the channel enhances and when $N \rightarrow 0$, the pressure is minimum. A similar observation is made for all values of $t$ ranging from $0-1$, i.e., throughout one time period. Temporal effects are similar to those observed for Newtonian and power-law fluids (cf. [13]). Figures, together with captions, provide the details (Figs. $2 \mathrm{a}-\mathrm{d}$ ).

We further carry out an investigation of the role of the other micro-polar parameter $M$. It is observed that the pressure along the entire length of the channel diminishes as $M$ increases. Hence, this parameter has an opposite influence vis-à-vis coupling number (Fig. 3).
Since no value of $M$ can lead to Newtonian nature, no comparison can be made with Newtonian fluids. In fact, the micro-polar fluid has a complex characteristic that is built up by the combined effects of these two parameters. This may be noticed that once $N=0$, $M$ seizes to affect the flow (cf. (17)).

Simultaneously, we consider the propagation of a non-integral number of waves in the train, which is an inherent feature of finite length vessels. Some significant differences are observed between this case and the other where there are an integral number of waves in the train. As a special case, we take $l=1.8$.

It is observed that unlike the case of an integral number of waves, the pressure distributions are different for the boluses enclosed within the whole and the fractional waves. Whatever be the temporal value, the pressure for the whole wave attains zero thrice: at the two ends and once midway depending upon the value of $t$ for the whole bolus while it reaches zero only twice at the two ends in the case of fractional bolus. But one 
(a)

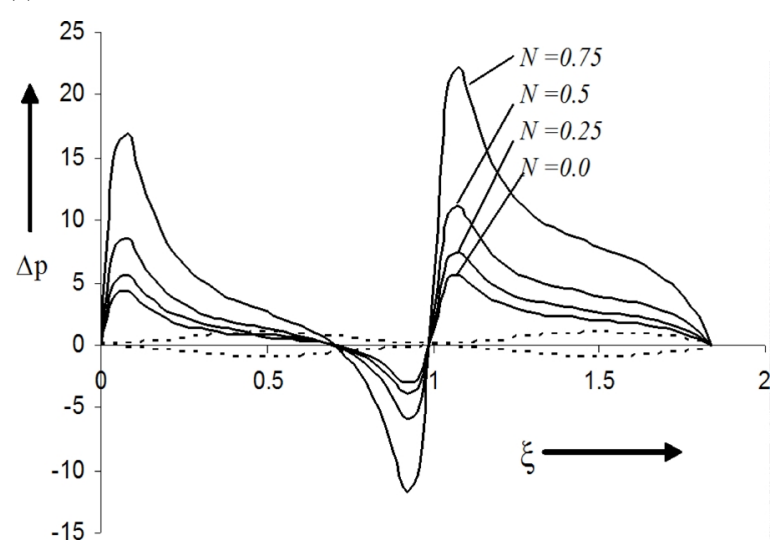

(c)

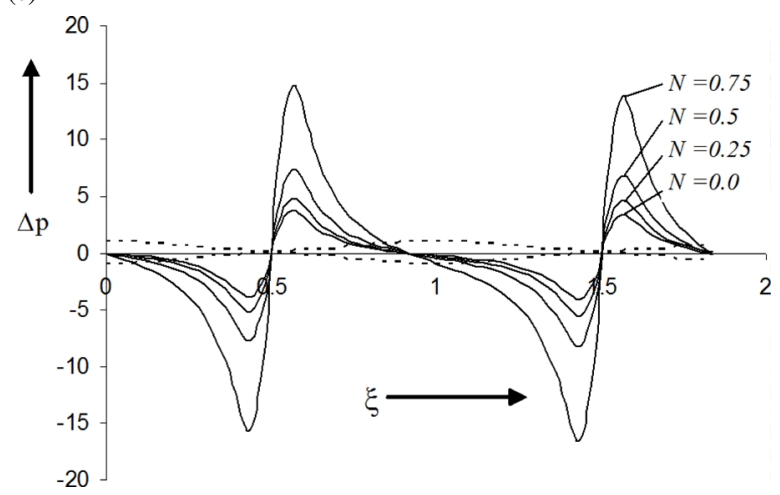

(b)

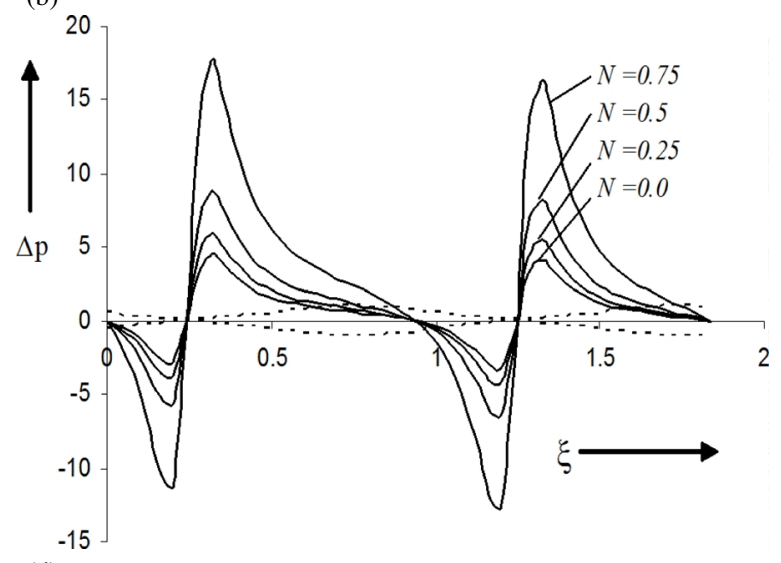

(d)

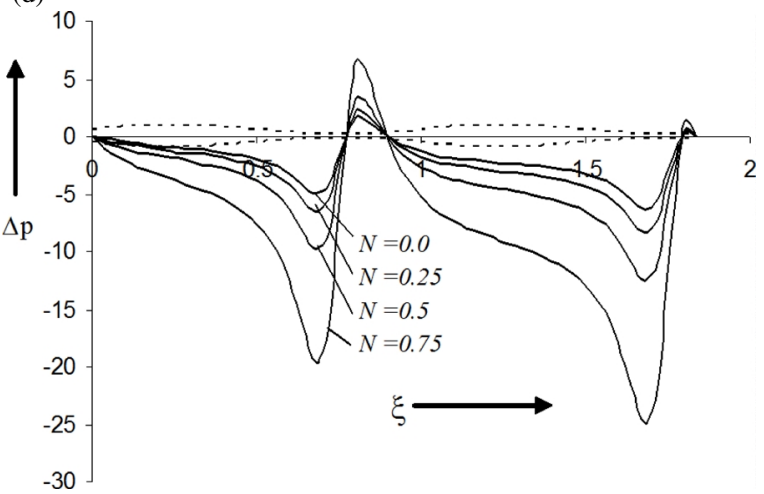

Fig. 4. Pressure vs. axial distance along the finite length channel at five time instants (a) $t=0.0, t=1.0$, (b) $t=0.25$, (c) $t=0.5$, (d) $t=0.75$. Dotted lines ( $\cdots$ ) represent the position of wave, whereas continuous lines $(-)$ represent the pressure distribution for different coupling number $N$ and for $\phi=0.9, l=1.8, M=1.0$. The pressures at the two ends are kept as zero.

significant difference between the two cases is that the peaks of pressure for the two different types of boluses are identical in the integral case while the peaks are different in the non-integral case (Fig. 4). Pressure distribution changes with $t$. It is further observed that the closer to symmetry is the distribution of boluses in the channel, the lesser is the difference between the pressure peaks (Fig. 3c). The effects of the coupling number $N$ and the micro-polar parameter $M$ do not reveal any difference in the two cases (Figs. 2-5).

A graph, plotted between pressure across one wavelength and averaged flow rate, that exhibits a linear relation between them, for varying coupling number $N$ and fixed $M$, indicates that the pressure increases with coupling number $N$. In fact, it measures the magnitude of pressure that can stop transportation. Therefore, once it is zero, maximum amount of flow can take place. For a fixed coupling number $N$, it is similar to that for Newtonian fluids (Fig. 6a). The obser- vation is that the pressure decreases with increasing $M$ and for fixed $N$ (Fig. 6b). Another point to note is that for a traditionally used sinusoidal wall equation that represents both contraction and expansion, comparatively more pressure is required to prevent the flow, that is to say, that the flow rate is less in the present case (Fig. 6c).

Figure 7a compares the two cases of unidirectional displacement of the wall and both ways of wall displacements. The quantitative differences are enormous. Graphs plotted for the mechanical efficiency reveal that it increases with the coupling number $N$ (Fig. 7b) for fixed $M$ and decreases with the micropolar parameter $M$ for fixed $N$ (Fig. 7c). Captions of the figures give details of the other parameters. The fluid turns Newtonian as $N \rightarrow 0$.

Reflux has been examined here only for small values of micro-polar parameter. The graphs based on (32) for reflux limit discloses that the reflux region for 
(a)

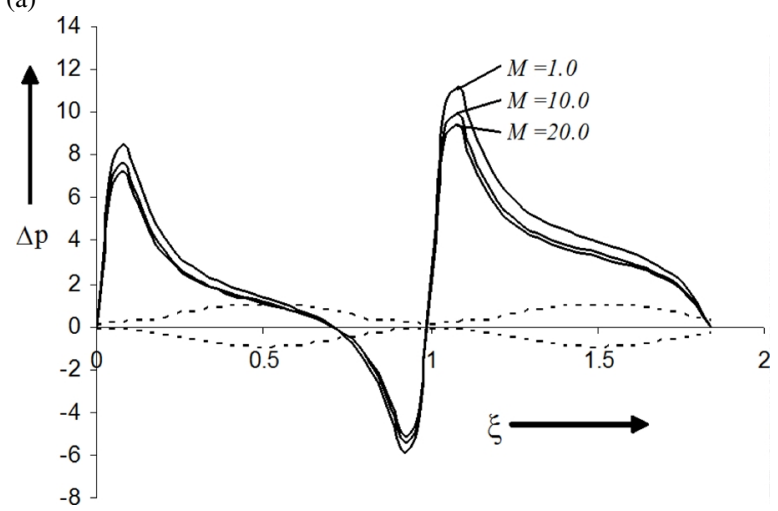

(c)

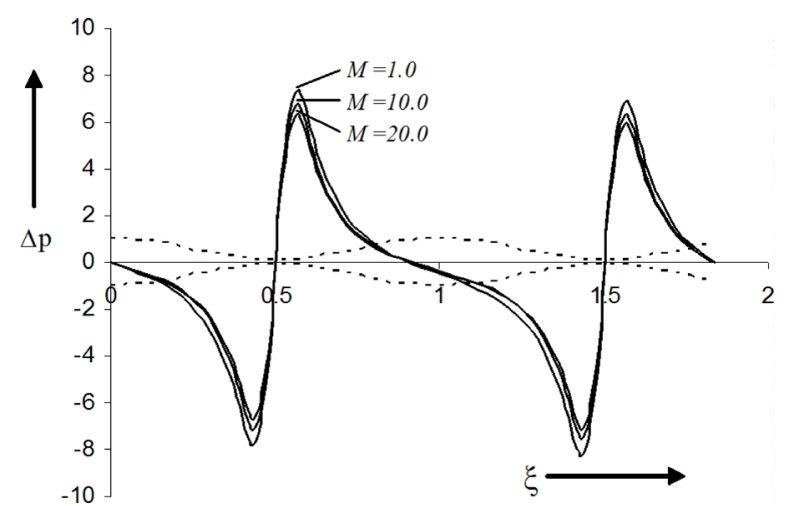

(b)

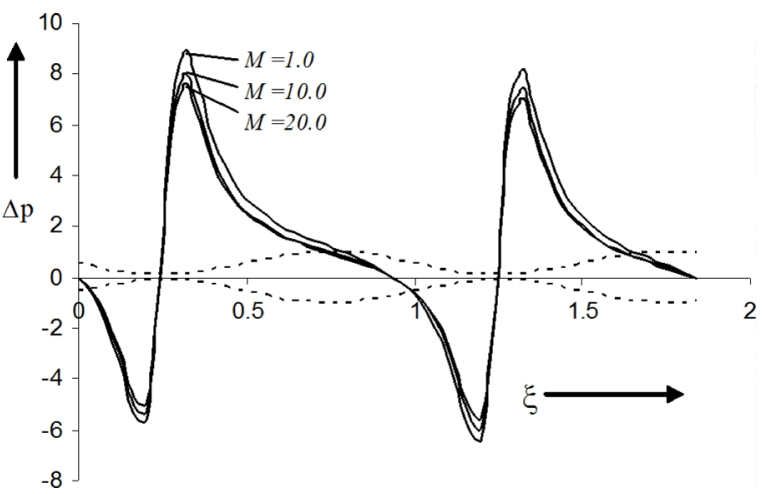

(d)

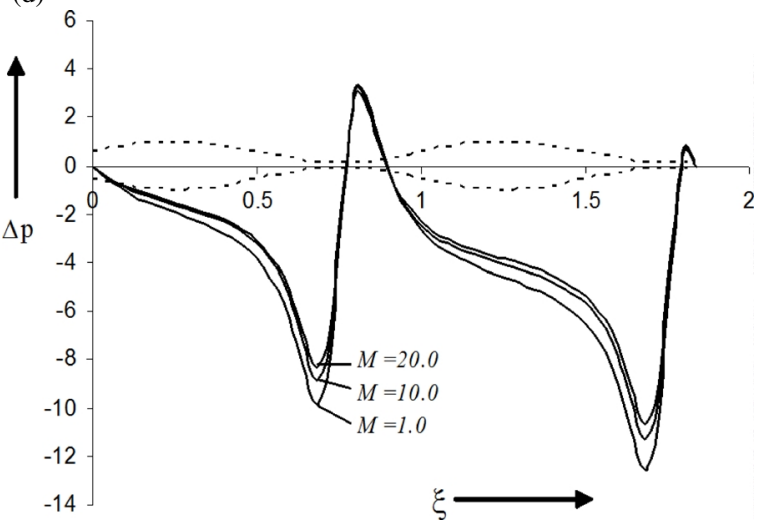

Fig. 5. Pressure vs. axial distance along the finite length channel at five time instants (a) $t=0.0, t=1.0$, (b) $t=0.25$, (c) $t=0.5$, (d) $t=0.75$. Dotted lines $(\cdots)$ represent the position of wave, whereas continuous lines ( -$)$ represent the pressure distribution for different micro-polar parameter $M$ and for $\phi=0.9, l=1.8, N=0.50$. The pressures at the two ends are kept as zero.

micro-polar fluid increases with the coupling number $N$ (Fig. 8a). Figure 8b compares the reflux regions for two cases of unidirectional displacement of the wall and both ways of wall displacements. It reveals that when the walls undergo contraction as well as expansion, the region favourable to reflux is significantly more than that in the present case. Since $M$ is considered very small, no significant difference is observed; and even if it is measured very carefully, it won't give us accurate values. For this reason, discussion in this regard is dropped.

Trapping (cf. [16]) has been studied for varying micro-polar parameter $M$ and coupling number $N$. We plot streamlines based on (27) for this case. Figures $9 \mathrm{a}-\mathrm{d}$ reveal that when $N$ is increased by keeping other parameters unchanged, the size of the trapped region decreases slightly. Unlike this, the size of the trapped region increases when $M$ enhances with other parameters remaining unaltered (Figs. 9c and e).

\subsection{Physical Interpretation and Applications in Biology and Engineering}

The observation of pressure distribution along the length of the channel leads to the conclusion that the mechanism is harmonic and works for a totally systematic continuous and rhythmic transportation and takes care of the pumping machinery for safety. In order to achieve this, the pressure distribution is time dependent. The case of integral number of waves suitably finds its application in oesophageal swallowing. Pressure distribution reveals that natural peristaltic pumping mechanism properly protects bolus movement from any possible retrograde motion of leading boluses and provides adequate safety to oesophagus. A further revelation is that swallowing of fluids resembling micropolar properties requires more efforts compared with Newtonian fluids such as water. This will be clear in subsequent paragraphs. 
footnotesize (a)

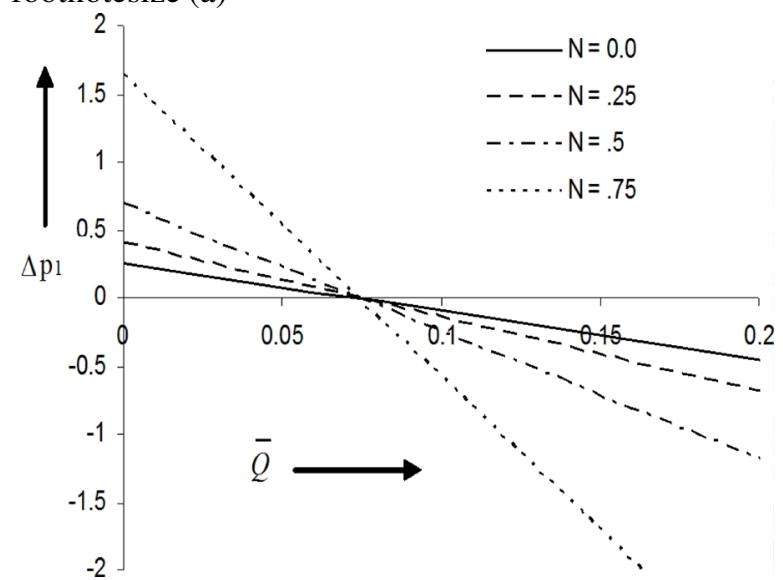

(c)

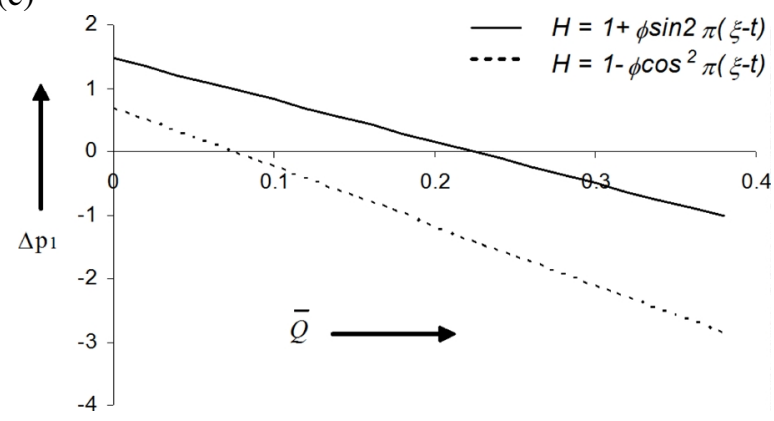

(b)

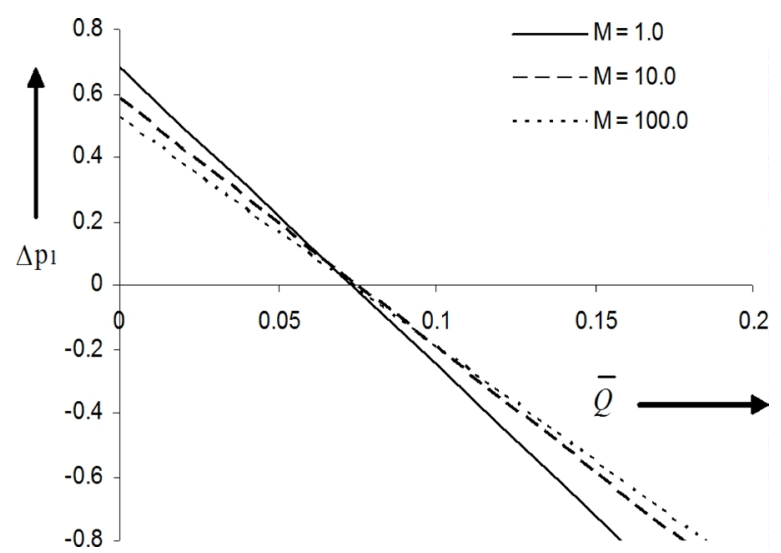

A non-integral number of waves is inherent to commercial pumps, so the related numerical results may be applied to this case. The observation in the case of non-integral number of waves propagating along the channel that the closer to symmetry the distribution of boluses in the vessel, the lesser is the difference between the pressure peaks, indicates that the difference in peak sizes of pressure is due to the positioning of waves. It may be inferred that pressure distribution in commercial pumps changes with the location of fractional waves and accordingly pressure peaks and troughs are unequal or almost equal. The pressure exerted by such a pump frequently changes in magnitude along its length.

It is found that pressure gradient as well as pressure along the length of the channel enhances as the coupling effect parameter $N$ increases. Since the coupling effect parameter $N$ is related with inner rotation, this may be physically interpreted as that the inner rotation of the fluid particles increases pressure; and as the fluid turns Newtonian, the pressure becomes minimum. This may lead to the conclusion that the channel has to work more to pump a micro-polar fluid.
Fig. 6. Pressure difference across one wavelength vs. average flow rate (a) for different coupling number $N$ and $\phi=0.4$, $M=1.0, l=1.0$, (b) for different micro-polar parameter $M$ and $\phi=0.4, N=0.50, l=1.0$, (c) for different wall equations and $\phi=0.4, N=0.50, M=1.0, l=1.0$.

The influence of the two parameters on the pressure across one wavelength and averaged flow rate disclose that micro-polar fluid requires more efforts to be transported.

This is noteworthy that while examining pumping efficiency, we fixed the flow rate so that higher efficiency is an indication that the pump has to be more efficient to transfer the same amount of micro-polar fluids. In other words, efficiency is less, in case a micropolar fluid is pumped.

The reflux region for micro-polar fluid increases with the coupling number. Physically it can be interpreted as that the flow becomes more prone to reflux when the inner rotation of fluid particles increases.

\section{Conclusion}

It is found that pressure distribution along the length of the channel is such that a bolus is very systematically carried away with the waves travelling along the walls of the channel. Micro-polar and Newtonian fluids have qualitatively similar pressure distributions; 
(a)

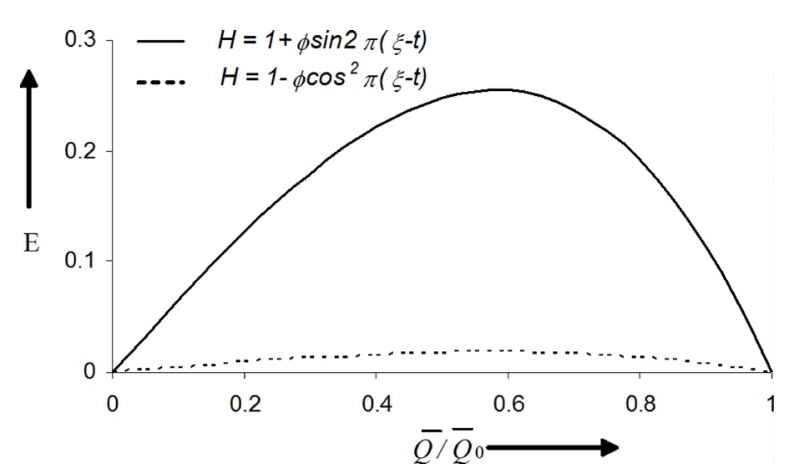

(c)

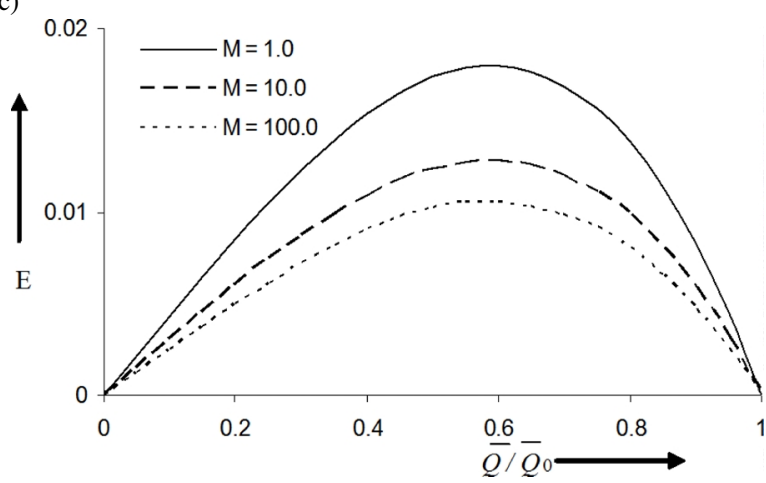

(a)

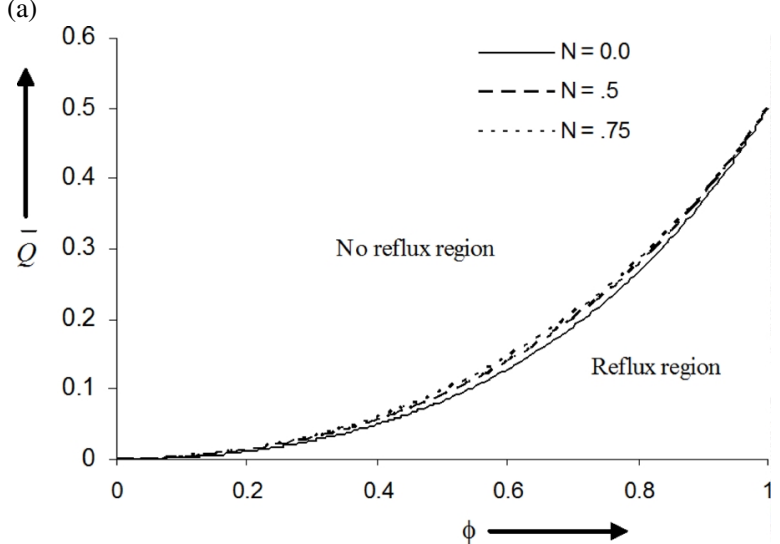

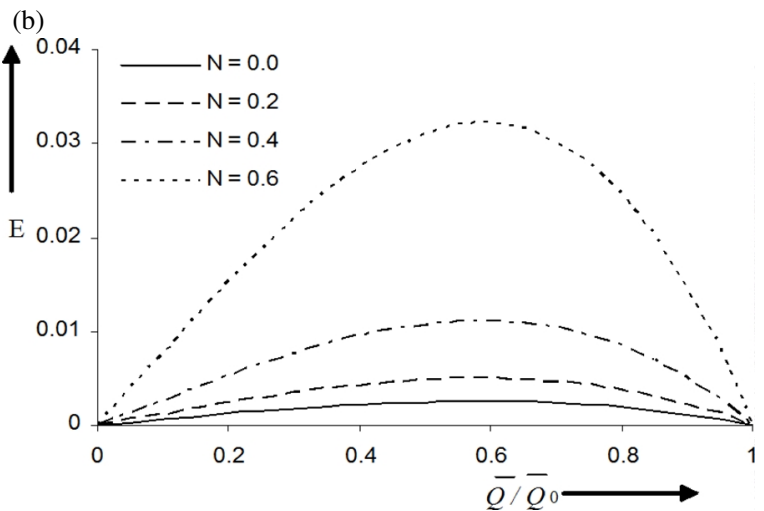

Fig. 7. Mechanical efficency vs. the ratio of average flow rate and maximum average flow rate relation (a) for different wall equations and $\phi=0.4, N=0.50, M=1.0$, (b) for corresponding coupling number $N$ and $\phi=0.4, M=1.0$, (c) for corresponding micro-polar parameter $M$ and $\phi=0.4$, $N=0.50$.

(b)

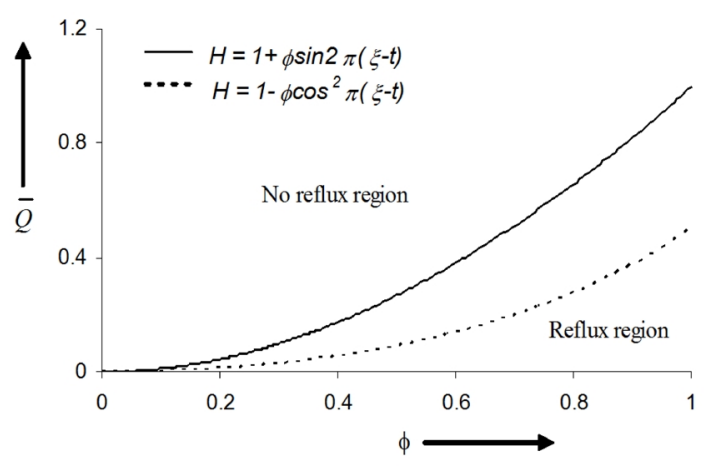

Fig. 8. Average flow rate vs. amplitude (a) for corresponding coupling number $N$ for $M=0.1$, (b) for different wall equations and $N=0.50, M=0.1$.

but the quantitative differences are significant enough. The parameters that represent the micro-polar character in a fluid influence the flow pattern much significantly. Revelation is that a parameter known as coupling number increases pressure along the entire length of the channel, while the other parameter decreases it. The latter one favours the flow, whereas the former one hinders it. The combined effect will be no doubt a complicated one. Had the exact experimental values of the two been available in the literature, we would have evaluated.

A pump with micro-polar fluid is also affected by the two parameters. The coupling number increases the efficiency; while the other micro-polar parameter de- 
(a)

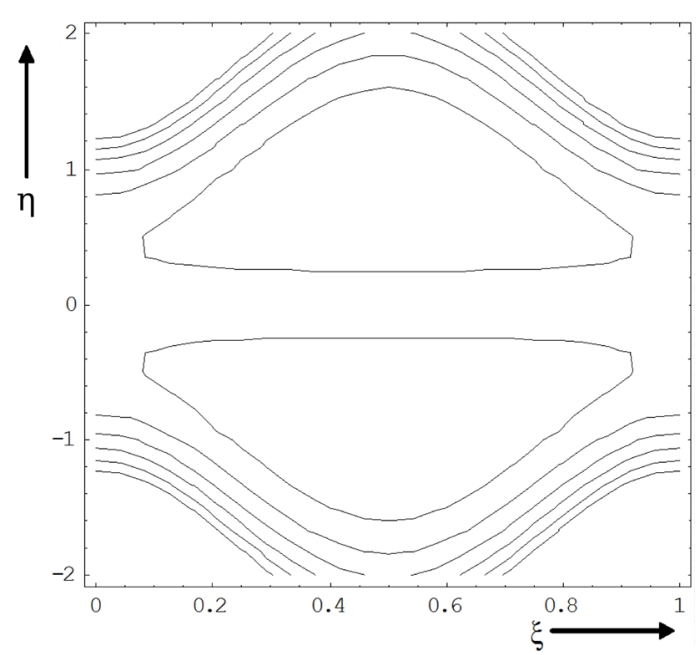

(c)

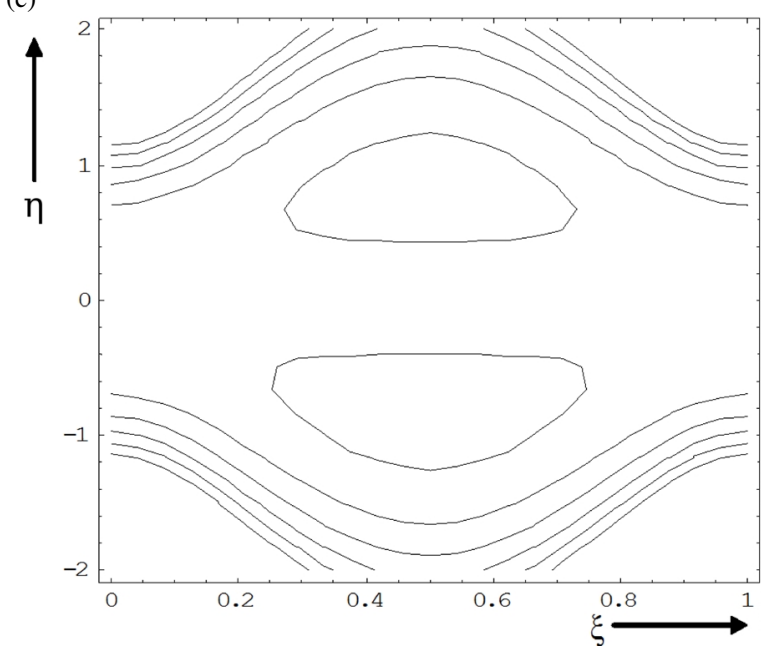

(e)

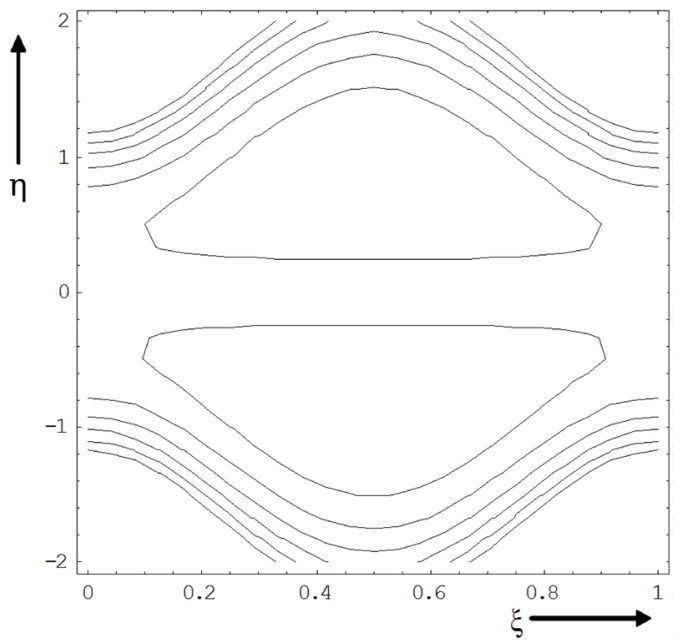

(b)

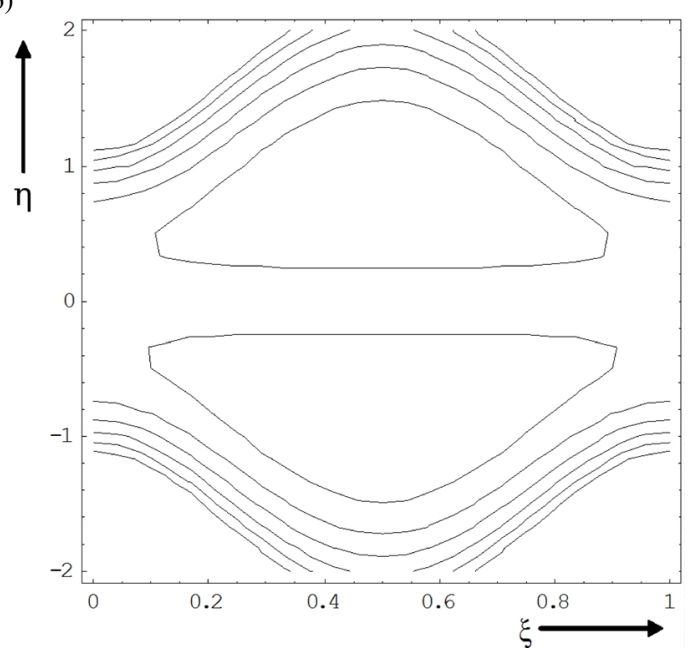

(d)

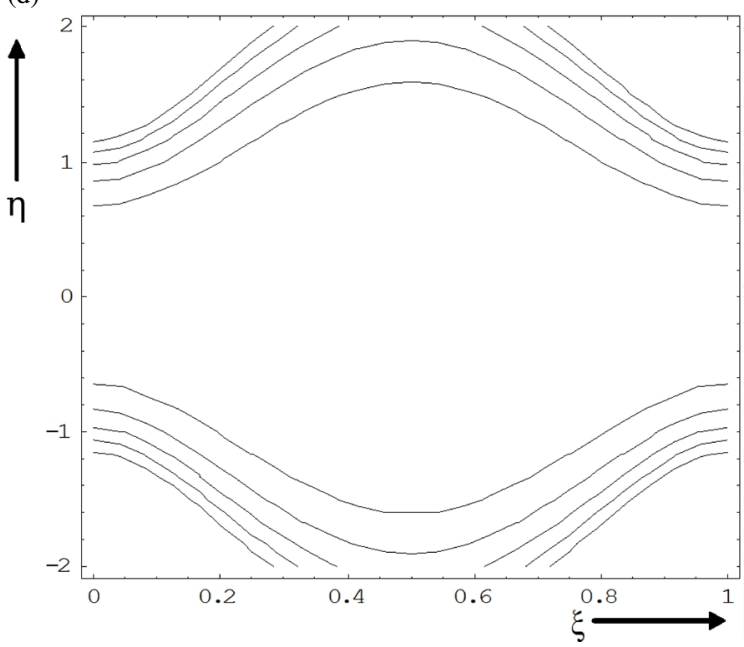

Fig. 9. Streamlines in the wave frame when (a) $\bar{Q}=0.6, \phi=$ $0.6, M=2.0, N=0.0$, (b) $\bar{Q}=0.6, \phi=0.6, M=2.0, N=$ 0.25 , (c) $\bar{Q}=0.6, \phi=0.6, M=2.0, N=0.5$, (d) $\bar{Q}=0.6$, $\phi=0.6, M=2.0, N=0.75$, (e) $\bar{Q}=0.6, \phi=0.6, M=5.0$, $N=0.5$. 
creases it. It is further concluded that the pumping efficiency decreases for micro-polar fluids.

Reflux region is found to increase with the coupling number. This may be inferred that a micro-polar fluid is more prone to flow reversal. One significant difference between integral and non-integral number of waves in the train propagating along the channel walls is that

[1] A. C. Eringen, J. Math. Mech. 16, 1 (1966).

[2] G. Devi and R. Devanathan, Proc. Indian Acad. Sci. A 81, 149 (1975).

[3] D. Philip and P. Chandra, Proc. Nat. Acad. Sci. India A 65, 63 (1995).

[4] R. Bhargava, S. Sharma, H. S. Takhar, T. A. Bég, O. A. Bég, and T. K. Hung, J. Biomech. 39, S649 (2006).

[5] D. Srinivasacharya, M. Mishra, and A. R. Rao, Acta Mech. 161, 165 (2003).

[6] T. Hayat, N. Ali, and Z. Abbas, Phys. Lett. A 370, 331 (2007).

[7] P. Muthu, B. V. R. Kumar, and P. Chandra, Appl. Math. Model. 32, 2019 (2008).

[8] T. Hayat and N. Ali, Math. Comput. Model. 48, 721 (2008).

[9] W. J. Dodds, Radiology of oesophagus, In A. R. Mar- the peaks of pressure are identical in the integral case while the peaks are different in the non-integral case. The effects of the coupling number and the micro-polar parameter are identical in both the cases of integral and non-integral number of waves in train.

Trapping is favoured by the coupling number but hindered by the micro-polar parameter.

gulis, H. J. Burhenne, (eds): Alimentary Tract Radiology, 3rd ed. 1983, 1529.

[10] J. Ren, B. T. Massey, W. J. Dodds, M. K. Kern, J. G. Brasseur, R. Shaker, S. S. Harrington, W. J. Hogan, and R. C. Arndorfer, Am. J. Physiol. 264, G407 (1993).

[11] M. Li and J.G. Brasseur, J. Fluid Mech. 248, 129 (1993).

[12] J. G. Brasseur and W.J. Dodds, Dysphagia 6, 100 (1991).

[13] J. C. Misra and S. K. Pandey, Math. Comput. Model. 33, 997 (2001)

[14] S. K. Pandey and D. Tripathi, Appl. Bionics Biomech. 7, 169 (2010).

[15] S. K. Pandey and D. Tripathi, J. Biol. Syst. 18, 621 (2010).

[16] A. H. Shapiro, M. Y. Jaffrin, and S.L. Weinberg, J. Fluid Mech. 35, 669 (1969). 